TRANSACTIONS OF THE

AMERICAN MATHEMATICAL SOCIETY

Volume 183, September 1973

\title{
ON FACTORIZED GROUPS
}

\author{
BY \\ DAVID C. BUCHTHAL $\left({ }^{1}\right)$
}

ABSTRACT. The effect on a finite group $G$ by the imposition of the condition that $G$ is factorized by each of its maximal subgroups has been studied by Huppert, Deskins, Kegel, and others. In this paper, the effect on $G$ brought about by the condition that $G$ is factorized by a normalizer of a Sylow $p$-subgroup for each $p \in \pi(G)$ is studied. Through an extension of a classical theorem of Burnside, it is shown that certain results in the case where the factors are maximal subgroups continue to hold under the new conditions. Definite results are obtained in the case where the supplements of the Sylow normalizers are cyclic groups of prime power order or are abelian Hall subgroups of $G$.

0 . Introduction. A well-known theorem of Huppert [6, p. 162] asserts that if every maximal subgroup of a finite group $G$ has prime index in $G$, then $G$ is supersolvable. The existence of the simple group of order 168 shows that the above theorem cannot be extended to the case where the maximal subgroups have prime-power index; nevertheless, a number of authors have obtained significant results by modifying the hypothesis and conclusion of Huppert's theorem.

Deskins [4] considered the case where the maximal subgroups had prime-power normal index. Kegel [13] investigated groups in which every maximal subgroup admitted a cyclic supplement of prime-power order. Later, other authors, such as Beidleman and Spencer [2], and Nyhoff [14] returned to the concept of normal index.

In the following, we take a different approach. Instead of looking at the index, we focus on the subgroups. Replacing maximal subgroups with Sylow normalizers, we prove that a finite group is solvable if each Sylow normalizer has prime-power index and go on to extend the corresponding result of Kegel.

All groups discussed are to be considered to be of finite order. Let $G$ be such a group. $\operatorname{Syl}_{p}(G)$ denotes the set of Sylow $p$-subgroups of $G, S_{p}$ an element of $\operatorname{Syl}_{p}(G), N_{G}\left(S_{p}\right)$ its normalizer in $G$, and $n_{p}(G)=\left|\operatorname{Syl}_{p}(G)\right|$. A group $N$ is a Sylow normalizer of $G$ if $N=N_{G}\left(S_{p}\right)$ for some $S_{p} \in \operatorname{Syl}_{p}(G)$ and $p \in \pi(G)$, the

Received by the editors April 3, 1972.

AMS (MOS) subject classifications (1970). Primary 20D40; Secondary 20D20.

Key words and phrases. Factorized group, Sylow subgroup, Sylow normalizer, cyclic supplements, abelian Hall supplements, solvable groups, supersolvable groups, Walter's theorem, Hall $\pi$-subgroup.

(1) The major part of this paper is a portion of the author's Ph. D. dissertation written at Purdue University under the direction of Professor Eugene Schenkman. The author spent a part of this time as a National Science Foundation Trainee. 
set of prime divisors of $|G|$. Recall that a Hall subgroup $H$ of $G$ is a subgroup whose order is relatively prime to its index in $G$. We write $H \stackrel{\simeq}{\leq} G$ to indicate that $H$ is isomorphic to a subgroup of $G$. The expression of $H \lessdot G$ denotes that $H$ is a maximal subgroup of $G$ and $H^{G}$ denotes the normal closure of $H$ in $G .|G|_{p}$ is the largest power of $p$ dividing $|G|$.

I. Sylow normalizers of prime-power index. The main result of this section is the following extension of a theorem of Burnside:

Theorem 1. If each Sylow normalizer of a group $G$ bas prime-power index in $G$, then $G$ is solvable.

Proof (Induction on $|G|$ ). The theorem is trivial in the case $|G|=1$. Suppose $|G|>1$ and the result true for all such groups of order less than $|G|$. By a result of M. Hall [7, p. 364], the hypothesis of the theorem is inherited by all factor groups of $G$ and normal subgroups of $G$.

We may therefore assume that $G$ is a nonabelian simple group. The proof will be completed by showing that no such simple group exists.

Let $p \in \pi(G)$ and suppose that $n_{p}(G)=r^{t}$. Then $r$ divides $n_{q}(G)$ for all $q \in \pi(G), q \neq r$. To see this, suppose the above statement false. Then there exists $q \in \pi(G)$ such that $r$ does not divide $n_{q}(G)$. Fix $S_{q} \in \operatorname{Syl}_{q}(G)$. Since $r$ does not divide $n_{q}(G)$, there exists some $S_{r} \in \operatorname{Syl}_{r}(G)$ such that $S_{r}<N_{G}\left(S_{q}\right)$. Thus $H=$ $S_{r} S_{q}=S_{q} S_{r}$ is a subgroup of $G$ with $S_{q} \triangleleft H$. Since $n_{p}(G)=r^{t}$, we can always find a Sylow $q$-subgroup of $G$ contained in a fixed $p$-Sylow normalizer of $G$. By Sylow's theorem, we may choose $S_{p} \in \operatorname{Syl}{ }_{p}(G)$ such that $S_{q}<N_{G}\left(S_{p}\right)$. Clearly $G=S_{r} N_{G}\left(S_{p}\right)$.

Since $S_{r}<H$, we have $G=H N_{G}\left(S_{p}\right)$. Thus all conjugates of $N_{G}\left(S_{p}\right)$ can be obtained by transforming $N_{G}\left(S_{p}\right)$ by elements of $H$. But $S_{q}<N_{G}\left(S_{p}\right)$ and $S_{q}<H$ imply that

$$
1 \neq S_{q} \leq \bigcap_{b \in H} N_{G}\left(S_{p}\right)^{b}=\bigcap_{g \in G} N_{G}\left(S_{p}\right)^{g} .
$$

Therefore, Core ${ }_{G}\left(N_{G}\left(S_{p}\right)\right)=\bigcap_{g \in G} N_{G}\left(S_{p}\right)^{g}$ is a nontrivial normal subgroup of $G$, contradicting the assumption that $G$ is simple.

We may thus assume that $r$ divides $n_{q}(G)$ for all $q \in \pi(G), r \neq q$. Suppose $n_{r}(G)=s^{v}$. Then $s \in \pi(G)$ and, by the same argument as above, $s$ divides $n_{q}(G)$ for all $q \in \pi(G), q \neq s$. By hypothesis, however, $n_{q}(G)$ is a prime power and we have shown that, for all $q \in \pi(G), q \neq r, q \neq s, n_{q}(G)$ is divisible by both $r$ and $s$. This can only happen if $\pi(G)$ contains just $r$ and $s$. Thus $|\pi(G)|=2$, and $G$ must be solvable by the Burnside Theorem, contradicting the nonabelian simplicity of $G$. Therefore, $G$ cannot exist, completing the proof of the theorem.

We may restate this result in terms of group factorizations. 
Corollary 1. Suppose $G$ is a group with the property that each Sylow normalizer of $G$ admits a supplement of prime-power order. Then $G$ is solvable.

Using Corollary 1, the author has been able to prove theorems similar to some results of O. H. Kegel [13]. Kegel studied the non-Frattini chief factors of a group in showing that $G$ is supersolvable if $G$ does not map homomorphically onto $\Sigma(4)$, the symmetric group on four letters, and every maximal subgroup of $G$ admits a supplement which is cyclic of prime power order.

In the case where $G$ is solvable, it is possible to replace "maximal subgroups" with "Sylow normalizers" and utilize a theorem of J. Ritt [17, p. 27], to show:

Theorem 2. Let $G$ be a solvable group with the property that every Sylow normalizer admits a cyclic supplement. Then $G$ is supersolvable or maps bomomorpbically onto $\Sigma(4)$.

This result has been generalized by David Perin [16]. The reader may consult Perin's paper for a proof of Theorem 2.

Combining Theorem 1 and Theorem 2, we obtain the following:

Corollary 2. Let $G$ be a group with the property that every Sylow normalizer is supplemented by a cyclic group of prime-power order. Then $G$ is supersolvable or $G$ maps onto $\Sigma(4)$.

II. Abelian Hall supplements. Kegel has conjectured that a group is solvable if each maximal subgroup admits an abelian supplement. The author feels that a similar statement can be made about the Sylow normalizers of a group. Corollary 2 is a proof in the case that the abelian supplements are cyclic of prime-power order.

In this last section, we show the solvability of finite groups with the property that each Sylow normalizer admits an abelian Hall supplement, i.e., a supplement which is also a Hall subgroup of $G$. The next result relies heavily upon the work of J. Walter concerning groups with abelian Sylow 2-subgroups.

Lemma 1. Let $G$ be a nonabelian group with the property that each Sylow normalizer admits an abelian Hall supplement. Then $G$ is nonsimple.

Proof. Suppose that $G$ is such a group and that $G$ is simple. Then $G=N_{p} A_{p}$ for all $p \in \pi(G)$, where $A_{p}$ denotes an abelian Hall supplement of $N_{p}$, a $p$-Sylow normalizer of $G$. If $N_{p} \cap A_{p} \neq 1$, for some $p \in \pi(G)$, then the normal closure of $N_{p} \cap A_{p}$ in $G$ is a nontrivial normal subgroup of $G$, contrary to our assumption that $G$ is simple. Therefore, $N_{p} \cap A_{p}=1$, for all $p \in \pi(G)$. Thus, $p \notin \pi\left(A_{p}\right)$ and $A_{p}$ is a Hall $\pi\left(N_{p}\right)^{\prime}$-complement in $G$. By the Feit-Thompson Theorem, we know that $2 \in \pi(G)$.

Consider $G=N_{2} A_{2}=N_{G}\left(S_{2}\right) A_{2}$ for some fixed $S_{2} \in \operatorname{Syl}_{2}(G)$ and some fixed $A_{2}$. Since $A_{2}$ is Hall, there exists some $S_{q} \in \operatorname{Syl}_{q}(G)$ such that $S_{q} \leq A_{2}$ and 
$\left|S_{q}\right| \neq 1$. Therefore, $A_{2} \leq N_{G}\left(S_{q}\right)$, since $A_{2}$ is abelian, and so

$$
N_{G}\left(S_{q}\right)=\left(N_{G}\left(S_{2}\right) \cap N_{G}\left(S_{q}\right)\right) A_{2} \text {. }
$$

Since $2 \notin \pi\left(A_{2}\right)$ and $A_{2} \cap\left(N_{G}\left(S_{2}\right) \cap N_{G}\left(S_{q}\right)\right) \leq A_{2} \cap N_{G}\left(S_{2}\right)=1$, a Sylow 2-subgroup of $N_{G}\left(S_{2}\right) \cap N_{G}\left(S_{q}\right)$ must be a Sylow 2-subgroup of $N_{G}\left(S_{q}\right)$. We consider two cases:

Case $1.2 \notin \pi\left(A_{q}\right)$, where $G=N_{G}\left(S_{q}\right) A_{q}$. Then $\left|S_{2}\right|=|G|_{2}=\left|N_{G}\left(S_{q}\right)\right|_{2}$, since $A_{q}$ is Hall, $N_{G}\left(S_{q}\right) \cap A_{q}=1$, and $2 \notin \pi\left(A_{q}\right)^{q}$. Hence, a Sylow 2-subgroup $D$ of $N_{G}\left(S_{2}\right) \cap N_{G}\left(S_{q}\right)$ is a Sylow 2-subgroup of $N_{G}\left(S_{q}\right)$. But $D \leq S_{2}$, since $S_{2}$ is the only Sylow 2-subgroup of $N_{G}\left(S_{2}\right)$. Therefore, $D=S_{2}$.

Let $g \in G, S_{q}^{g} \neq S_{q}$. Then $g=a n, a \in A_{2}, n \in N_{G}\left(S_{2}\right)$ and $S_{q}^{g}=S_{q}^{n}$, since $S_{q} \unlhd A_{2}$. But then $S_{2} \leq N_{G}\left(S_{q}\right)$ implies $S_{2}=S_{2}^{n} \leq N_{G}\left(S_{q}\right)^{n}=N_{G}\left(S_{q}^{n}\right)=N_{G}\left(S_{q}^{g}\right)=$ $N_{G}\left(S_{q}\right)^{g}$. Since $S_{q}^{g}$ was an arbitrary conjugate of $S_{q}$, Sylow's theorem implies that $S_{2}$ is contained in every conjugate of $N_{G}\left(S_{q}\right)$. Therefore, $S_{2}$ is contained in Core ${ }_{G}\left(N_{G}\left(S_{q}\right)\right)$, a normal subgroup of $G$, and $G$ is nonsimple.

Case 2. $2 \in \pi\left(A_{q}\right)$. Then $S_{2}$ is abelian, since $A_{q}$ is an abelian Hall subgroup. By the classification theorem of J. Walter [23, p. 405] on groups with abelian Sylow 2-subgroups, we have that $G$ is isomorphic to one of the following groups:

(i) $\operatorname{PSL}\left(2,2^{n}\right), n>1$.

(ii) PSL $\left(2, p^{n}\right), p^{n} \equiv 3$ or $5(\bmod 8), p^{n}>3$.

(iii) A simple group $M$ such that for each involution $j$ of $M, C_{M}(j)=\langle j\rangle \times K$, where $K \cong \operatorname{PSL}(2, r), r \equiv 3$ or $5(\bmod 8)$.

We will show that none of the above groups satisfies the hypothesis of our theorem. This will complete the proof of the theorem, since we will have then shown that Case 2 cannot occur.

The possible factorizations of the projective special linear groups PSL $\left(2, p^{n}\right)$ have been determined by N. Ito [10]. For completeness, we outline a direct argument. In the following discussion of the projective special linear groups, we will make use of many properties of such groups and omit the page references to each property used, referring the reader to [9, Chapter II, \$8].

Suppose $G \cong \operatorname{PSL}\left(2,2^{n}\right), n>1$. Let $p \in \pi(G), p \neq 2$. Since $|G|=$ $\left(2^{n}+1\right) 2^{n}\left(2^{n}-1\right)$, we have that $p$ divides $2^{n}+1$ or $2^{n}-1$, but not both. In the first case, $G$ possesses a cyclic subgroup of order $2^{n}+1$ and $N_{G}\left(S_{p}\right)$ is a dihedral group of order $2\left(2^{n}+1\right)$. Since $A_{p}$ has been assumed to be an abelian Hall-complement for all $p \in \pi(G), 2 \notin \pi\left(A_{p}\right)$. In the second case, a similar argument shows that $2 \notin \pi\left(A_{p}\right)$. The choice of $p$ was arbitrary; thus, $2 \notin \pi\left(A_{p}\right)$ for any odd $p \in \pi(G)$. In particular, $2 \notin \pi\left(A_{q}\right)$, contrary to assumption. Hence, if $G$ is simple and satisfies our hypothesis, $G \nsubseteq \operatorname{PSL}\left(2,2^{n}\right)$.

Suppose $G \cong \operatorname{PSL}\left(2, p^{n}\right), p^{n} \equiv 3$ or $5(\bmod 8), p^{n}>3$. Then a Sylow $p$-subgroup of $G$ is elementary abelian and $n_{p}(G)=p^{n}+1$. Therefore, the order of the 
normalizer of a Sylow $p$-subgroup is $p^{n}\left(p^{n}-1\right) / 2$. Since 2 divides $p^{n}+1$, our conditions force $p^{n}\left(p^{n}-1\right) / 2$ and hence $\left(p^{n}-1\right) / 2$ to be odd. Let $t$ be an odd prime factor of $p^{n}-1$. The existence of such a $t$ is assured, since $p^{n}>3$. As above, $t$ does not divide $p^{n}+1$, and if $S_{t} \in \operatorname{Syl}_{t}(G)$, then $\left|S_{t}\right|$ divides $p^{n}-1$. Now $G$ contains a cyclic subgroup $L$ of order $\left(p^{n}-1\right) / 2$ with the property that $N_{G}\left(S_{t}\right)$ is a dihedral group of order $2|L|=p^{n}-1$. But then $\left(p^{n}+1\right) / 2$ is odd, forcing $|G|_{2}=2$ and $G$ to be nonsimple by [18, p. 139].

We have reduced to the case (iii). If $r \equiv 5(\bmod 8)$, then $M$ has been shown to be $J(11)$, the group of order 175,560 discovered by Janko [11]. $J(11)$ has a 7 Sylow normalizer of order 42 and index 4,180 . Therefore, $G \geqq J(11)$, since $J(11)$ cannot contain a Hall complement of its 7 -Sylow normalizer. Thus, $r \not \equiv 5(\bmod 8)$.

In the case $r \equiv 3(\bmod 8)$, Walter [22], [23], Janko and Thompson [12], and Ward [21] have determined a great deal of information about $M$, known as a group of Ree-type. Here $M$ has order $r^{3}\left(r^{3}+1\right)(r-1)$, and $r=3^{2 n+1}, n>0$. Moreover, if $S_{3} \in \operatorname{Syl}_{3}(M)$, then $\left|S_{3}\right|=r^{3}$, with $\left|N_{M}\left(S_{3}\right)\right|=r^{3}(r-1)$. Now the fact that $r$ is odd implies that $r-1$ and $r^{3}+1$ are even. Thus a 3-Sylow normalizer of $M$ cannot have a Hall-complement; hence $G \nsubseteq M$.

In disposing of the above cases, we have shown that Case 2 cannot occur and thus that $G$ is nonsimple.

Theorem 3. Let $G$ be a group in which every Sylow normalizer admits an abelian Hall supplement. Then $G$ is solvable.

Proof (Induction on $|G|$ ). We may assume $|G|>1$ and the result true for all such groups $H$ for which $|H|<|G|$. By Lemma 1 , if $G$ is nonabelian, then $G$ is nonsimple. Let $T$ be a normal subgroup of $G$. Then, for each $p \in \pi(G / T)$,

$$
G / T=\left(N_{G}\left(S_{p}\right) T / T\right)\left(A_{p} T / T\right)=\left(N_{G / T}\left(S_{p}\right) T / T\right)\left(A_{p} T / T\right)
$$

[9, p. 35], where $G=N_{G}\left(S_{p}\right) A_{p}, S_{p} \in \operatorname{Syl}_{p}(G)$. Since $p \in \pi(G / T), S_{p} T / T \epsilon$ $\operatorname{Syl}_{p}(G / T)$ and $N_{G / T}\left(S_{p} T / T\right)$ is supplemented (possibly trivially) by $A_{p} T / T$. Now $A_{p} T / T$ is an abelian Hall subgroup of $G / T$, for $A_{p} T / T$ is a $\pi\left(A_{p}\right)$-group and $\left[G / T: A_{p} T / T\right]=\left[G: A_{p} T\right]$ divides $\left[G: A_{p}\right]$, a $\pi\left(A_{p}{ }_{p}\right)^{\prime}$-number. Thus, every nontrivial factor group of $G$ is solvable by our induction hypothesis.

We suppose that $G$ is nonsolvable and show a contradiction in a series of steps.

(1) $G$ contains no nontrivial normal solvable subgroup.

If $T$ is such a group, then by the above, $G / T$ is solvable and hence, $G$ is solvable.

(2) $G$ has a unique minimal normal subgroup $K$ and $C_{G}(K)=1$.

If $K$ and $K_{0}$ are distinct minimal normal subgroups of $G$, then $G \cong G /\left(K \cap K_{0}\right)$ $\approx G / K \times G / K_{0}$, forcing $G$ to be solvable by induction. Therefore, $K$ is unique. If $\phi(G) \neq 1$, then $G / \phi(G)$ is solvable by induction, implying $G$ is solvable. Thus, $\phi(G)=1$ and there exists $M<G$ such that $G=M K$ and Core ${ }_{G}(M)=1$. The statement follows from a result of Baer [1, p. 119]. 
(3) $G=N_{G}\left(S_{p} \cap K\right) K$ for all $p \in \pi(G), S_{p} \in \operatorname{Syl}_{p}(G)$.

This follows by the Frattini argument [18, p. 129].

(4) $K \underline{\not} N_{p}$ for any $p \in \pi(K)$.

Suppose $p \in \pi(K)$ and $K \leq N_{p}=N_{G}\left(S_{p}\right)$, for some $S_{p} \in \operatorname{Syl}_{p}(G)$. Then $N_{p} \leq$ $N_{G}\left(S_{p} \cap K\right)$ and $S_{p} \cap K \neq 1$, forcing a contradiction by (3) and (1).

(5) $N_{p} \cap A_{p}=1$ for all $p \in \pi(K)$, where $G=N_{p} A_{p}$.

If $D=N_{p} \cap A_{p} \neq 1$, then $D^{G}$ is contained in $N_{p}$, forcing $K$ to be contained in $N_{p}$ by the uniqueness of $K$ and contradicting (4).

(6) $G=N_{G}\left(S_{p}\right) K$ for all $p \in \pi(K), S_{p} \in \operatorname{Syl}_{p}(G)$.

We show that $N_{G}\left(S_{p}\right)=N_{G}\left(S_{p} \cap K\right)$ for all $p \in \pi(K), S_{p} \in \operatorname{Syl}_{p}(G)$. The result then follows from (3). Clearly, $N_{G}\left(S_{p}\right) \leq N_{G}\left(S_{p} \cap K\right)$, and so $G=N_{G}\left(S_{p} \cap K\right) A_{p}$, where $G=$ $N_{G}\left(S_{p}\right) A_{p}$. If the containment is proper, then $N_{G}\left(S_{p} \cap K\right) \cap A_{p} \neq 1$. Therefore, $\left(N_{G}\left(S_{p} \cap K\right) \cap A_{p}\right)^{G}$ is a nonerivial normal subgroup of $N_{G}\left(S_{p} \cap K\right)$, forcing $K$ to be contained in $N_{G}\left(S_{p} \cap K\right)$. But then, $S_{p} \cap K$ is a solvable normal subgroup of $G$ by (3), contradicting (1).

(7) $|K|=\left|N_{K}\left(S_{p}\right)\right| \cdot\left|A_{p}\right|$ for all $p \in \pi(K)$, where $G=N_{G}\left(S_{p}\right) A_{p}$, and $S_{p} \in \operatorname{Syl}_{p}(G)$.

We have

$$
\begin{aligned}
{\left[K: N_{K}\left(S_{p}\right)\right] } & =\left[K: N_{G}\left(S_{p}\right) \cap K\right]=\left[K N_{G}\left(S_{p}\right): \bar{N}_{G}\left(S_{p}\right)\right] \\
& =\left[G: N_{G}\left(S_{p}\right)\right] \text { by }(6) \\
& =\left|A_{p}\right| \quad \text { by }(5) .
\end{aligned}
$$

Therefore, $|K|=\left|N_{K}\left(S_{p}\right)\right| \cdot\left|A_{p}\right|$.

(8) If $G=N_{p} A_{p}$ for $p \in \pi(K)$, then $A_{p} \leq K$.

Now, $A_{p} \cap K \in \mathrm{Hall}_{\pi\left(A_{p}\right)}(K)$. But the above paragraph implies that $|K|_{\pi\left(A_{p}\right)}=$ $\left|A_{p}\right|$. Therefore, $A_{p} \cap K=A_{p}$, and $A_{p} \leq K$.

(9) $K=N_{K}\left(S_{p} \cap K\right) A_{p}$ for all $p \in \pi(K), S_{p} \in \operatorname{Syl}_{p}(G)$, where $G=N_{G}\left(S_{p}\right) A_{p}$.

Since $A_{p} \leq K$ and $K \leq N_{G}\left(S_{p}\right) A_{p}=G$, the Dedekind Lemma [9, p. 8] implies that $K=\left(N_{G}\left(S_{p}\right) \cap K\right) A_{p}$. Since $N_{G}\left(S_{p}\right) \cap K=N_{K}\left(S_{p} \cap K\right)$ by the proof of (6), $K=N_{K}\left(S_{p} \cap K\right) A_{p}$.

If $K=G$, then $G$ would be simple, contrary to Lemma 1 , and the assumption that $G$ is not solvable and, hence, nonabelian. Therefore, $K$ is a proper subgroup of $G$ which, by (9), has the property that each of its Sylow normalizers admits an abelian Hall supplement. Thus, $K$ is solvable by induction.

This last step is the desired contradiction. By (1), $K$ cannot be solvable but, by (9), $K$ must be solvable. Therefore, $G$ must be solvable.

Corollary 3. Let $G$ be a group in which every Sylow normalizer admits a cyclic Hall supplement. Then $G$ is supersolvable. 
Proof. By Theorem 3, $G$ is solvable. Theorem 2 then implies that $G$ is supersolvable or $G$ maps onto $\Sigma(4)$. We show that the latter cannot occur.

As in the proof Theorem 3, the hypothesis carries over to all factor groups of $G$. If $G$ were to map onto $\Sigma(4)$, then a 3-Sylow normalizer of $\Sigma(4)$ would be supplemented by a cyclic Hall subgroup of order 8 . But the Sylow 2-subgroups of $\Sigma(4)$ are noncyclic. The result follows.

\section{BIBLIOGRAPHY}

1. R. Baer, Classes of finite groups and their properties, Illinois J. Math. 1 (1957), 115-187. MR 19, 386.

2. J. Beidleman and A. Spencer, The normal index of maximal subgroups in finite groups, Illinois J. Math. 16 (1972), 95-101.

3. S. A. Čunihin, Subgroups of finite groups, Nauka i Tehnika, Minsk, 1964; English transl., Wolters-Noordhoff, Groningen, 1969. MR 35\#2957; $40 \# 1463$.

4. W. E. Deskins, On maximal subgroups, Proc. Sympos. Pure Math., vol. 1, Amer. Math. Soc., Providence, R. I., 1959, pp. 100-104. MR 23 \#A2462.

5. W. Feit and J. G. Thompson, Solvability of groups of odd order, Pacific J. Math. 13 (1963), 775-1029. MR 29 \#3538.

6. M. Hall, Jr., The theory of groups, Macmillan, New York, 1959. MR 21 \#1996.

7. - On the number of Sylow subgroups in a finite group, J. Algebra 7 (1967), 363-371. MR $36 \# 5211$.

8. P. Hall, A characteristic property of solvable groups, J. London Math. Soc. 12 (1937), 188-200.

9. B. Huppert, Endliche Gruppen.I, Die Grundlehren der math. Wissenschaften, Band 134, Springer-Verlag, Berlin and New York, 1967. MR 37 \#302.

10. N. Ito, On the factorizations of the linear fractional group $L F\left(2, p^{n}\right)$, Acta. Sci. Math. Szeged 15 (1953), 79-84. MR 15, 287.

11. Z. Janko, A new finite simple group with abelian Sylow 2-subgroups and its characterization, J. Algebra 3 (1966), 147-186. MR $33 \# 1359$.

12. Z. Janko and J. G. Thompson, On a class of finite simple groups of Ree, J. Algebra 4 (1966), 274-292. MR 34 \#1386.

13. O. H. Kegel, On Huppert's characterization of finite supersolvable groups, Proc. Internat. Conf. Theory of Groups (Canberra, 1965), Gordon and Breach, New York, 1967, pp. 209-215. MR $36 \# 274$.

14. L. R. Nyhoff, The influence on a finite group of the cofactors and subcofactors of its subgroups, Trans. Amer. Math. Soc. 154 (1971), 459-491. MR $44 \# 1720$.

15. O. Ore. Contributions to the theory of groups of finite order, Duke Math. J. 5 (1938), 431-460.

16. David Perin. Finite groups with nicely supplemented Sylow normalizer, Trans. Amer. Math. Soc. 183 (1973), 433-437.

17. J. F. Ritt, On algebraic functions which can be expressed in terms of radicals, Trans. Amer. Math. Soc. 24 (1923), 21-30.

18. E. Schenkman, Group theory, Van Nostrand, Princeton, N. J., 1965. MR 33 \#5702.

19. W. R. Scott, Group theory, Prentice-Hall, Englewood Cliffs, N. J., 1964. MR 29 \#4785. 
20. J. Szep and L. Rédei, On factorisable groups, Acta Univ. Szeged. Sect. Sci. Math. 13 (1950), 235-238. MR 14, 13, 1277.

21. H. N. Ward, On Ree's series of simple groups, Trans. Amer. Math. Soc. 121 (1966), 62-89. MR 33 \#5752.

22. J. H. Walter, Finite groups with abelian Sylow 2-subgroups of order 8, Invent. Math. 2 (1967), 332-376. MR 36 \#1531.

23. - The characterization of finite groups with abelian Sylow 2-subgroups, Ann. of Math. (2) 89 (1969), 405-514. MR 40 \#2749.

DEPARTMENT OF MATHEMATICS, UNIVERSITY OF AKRON, AKRON, OHIO 44325 